\title{
Overexpression of LBX2 associated with tumor progression and poor prognosis in colorectal cancer
}

\author{
XIAODONG HUANG, YUJIE YANG, CHAO YANG, HUALI LI, HUANGRONG CHENG and YONGBIN ZHENG
}

Department of Gastrointestinal Surgery, Renmin Hospital of Wuhan University, Wuhan, Hubei 430060, P.R. China

Received September 1, 2019; Accepted January 24, 2020

DOI: 10.3892/ol.2020.11489

\begin{abstract}
Colorectal cancer (CRC) is one of the most common carcinomas with high morbidity and mortality worldwide. However, the underlying molecular mechanisms of CRC are unclear. The aim of the present study was to establish the role that overexpression of LBX2 serves in CRC and to investigate the associated biological pathways. The difference in the expression levels of LBX2 between CRC tissues and adjacent normal colorectal tissues was assessed using the Oncomine database and Tumor Immune Estimation Resource. The expression levels of LBX2 and its prognostic significance in CRC were analyzed using a t-test and $\chi^{2}$ test using data from The Cancer Genome Atlas database. The Kaplan-Meier method and Cox regression analysis were used to estimate the prognostic value of LBX2 in CRC. Furthermore, the potential mechanisms of LBX2 dysregulation and its underlying molecular mechanisms in CRC were investigated using Gene Set Enrichment Analysis (GSEA). LBX2 expression levels were significantly upregulated in CRC samples compared with corresponding normal colorectal tissues $(\mathrm{P}<0.05)$. LBX2 upregulation was correlated with advanced tumor stage (III or IV), vascular invasion, lymphatic invasion and the male sex (all $\mathrm{P}<0.05)$. Kaplan-Meier analyses showed that high expression levels of LBX2 were associated with a less favorable overall survival (OS) and disease-free survival (DFS) in CRC (all $\mathrm{P}<0.05$ ). Multivariate analyses further confirmed that LBX2 upregulation was an independent indicator of less favorable OS and DFS (all $\mathrm{P}<0.05$ ). In addition, LBX2 DNA hypomethylation and microRNA (miR)-378a-3p downregulation correlated with LBX2 upregulation in CRC (all $\mathrm{P}<0.05$ ). The downregulation of miR-378a-3p in CRC was also significantly associated with less favorable OS and DFS, as demonstrated using Kaplan-Meier analyses (all $\mathrm{P}<0.05$ ). Moreover, GSEA indicated that 'VEGF signaling', 'Cell adhesion molecules CAMs', 'Toll-like receptor signaling'
\end{abstract}

Correspondence to: Professor Yongbin Zheng, Department of Gastrointestinal Surgery, Renmin Hospital of Wuhan University, 99 Zhangzhidong Road, Wuhan, Hubei 430060, P.R. China

E-mail: 13871189698@163.com

Key words: colorectal cancer, LBX2, miR-378a-3p, prognosis and 'Natural killer cell-mediated cytotoxicity' signaling pathways were enriched in the high LBX2 expressing cohort (all $\mathrm{P}<0.05$ ). Thus, overexpression of LBX2 may be associated with the development of CRC and may serve as a novel prognostic marker and therapeutic target in CRC. The mechanisms of LBX2 upregulation in CRC are possibly associated with LBX2 DNA hypomethylation and miR-378a-3p downregulation. The potential mechanisms of LBX2 upregulation in CRC might be regulated via the 'Cell adhesion molecules CAMs', 'Toll-like receptor signaling' and 'Natural killer cell-mediated cytotoxicity' signaling pathways.

\section{Introduction}

In 2018, colorectal cancer (CRC) was reported to be the third most commonly diagnosed malignancy and the second leading cause of cancer-associated mortalities worldwide (1). Although many advances in the early detection and comprehensive therapy of CRC have prolonged the survival time of patients with CRC, patients with advanced CRC in the United States have been reported to still experience tumor progression, relapse and metastasis following initial surgery (2). Several studies have demonstrated that complex genomic and epigenomic alterations that represent the heterogeneous molecular characterization of CRC are the leading causes of tumorigenesis and progression of CRC (3-5). In addition to immune response and influences by exogenous factors, the complexity of molecular genetic mechanisms remains a challenge to current personalized therapeutic approaches to CRC (6). Therefore, it is important to identify genes associated with the pathogenesis and prognosis of CRC to find candidate molecular biomarkers and therapeutic targets.

LBX2, located on chromosome 2p13.1, is a transcription factor gene with barely any previous functional studies or known animal models (7). LBX2 serves a role in body pattern formation as well as morphogenesis of the heart, skeletal muscle and nervous systems in vertebrates, such as zebrafish and mice (7). In zebrafish, LBX2 has been reported to regulate gastrulation movements and hypaxial myogenesis through the canonical or noncanonical Wnt/ $\beta$-catenin signaling pathway $(8,9)$. Owing to its diversity in functional domains, previous studies have revealed that LBX2 is not only involved in diverse physiological processes, including the regulation of myofibril formation and development of heart $(7,10)$, but also tumorigenesis. For example, the aberrant expression of LBX2 has been observed 
in several types of cancers, such as non-small cell lung cancer, adenoid cystic carcinoma and T-cell acute lymphoblastic leukemia (11-13). The methylation and downregulation of LBX2 are also associated with adenoid cystic carcinoma development and progression (12). Thus, LBX2 deregulation may contribute to carcinogenesis; however, the expression levels of LBX2 and its role in CRC remain unclear.

To investigate the significance of LBX2 expression in $\mathrm{CRC}$, a comprehensive analysis of the association between the mRNA expression levels of LBX2 and prognosis in CRC was conducted by mining data from The Cancer Genome Atlas (TCGA) database (cancer.gov/tcga). Furthermore, to investigate the potential underlying molecular mechanisms of LBX2 dysregulation and corresponding biological pathways in CRC, the regulatory miRNAs of LBX2 were evaluated and Gene Set Enrichment Analysis (GSEA) was performed to find potential signaling pathways associated with the upregulation of LBX2 in CRC. The present study is the first to describe the deregulation of LBX2 in TCGA colon adenocarcinoma and rectum adenocarcinoma cohorts of 383 patients with $\mathrm{CRC}$ and the association between LBX2 expression levels and survival.

\section{Materials and methods}

Mining data from TCGA database. The gene expression data of mRNA sequencing, miRNA sequencing and the corresponding clinical datasheets of 51 normal colorectal issues and 383 primary CRC samples were obtained from the TCGA website. Both the mRNA sequencing data and clinical information of all 383 patients with CRC and the miRNA sequencing data of 324 patients were analyzed. Sequenced data were downloaded using the Illumina HiSeq_RNA-Seq and Illumina HiSeq_miRNA-Seq platforms (portal.gdc.cancer.gov). The $\mathrm{R}$ software package 'edgeR' (version 3.6.0; R-project.org) was used to normalize and process the downloaded data and identify the differentially expressed genes between the CRC samples and normal controls. $\log _{2}$ conversions were performed for all gene expression data. The threshold was determined according to the following values: $\log _{2}$ FoldChange $>1$ and adjusted P-value $<0.05$. The gene-expression levels of LBX2 in CRC was reviewed using the Oncomine database (oncomine.org/resource/login.html) and Tumor Immune Estimation Resource (TIMER) (cistrome.shinyapps.io/timer/). The LBX2 DNA copy number alterations (CNA) and methylation data were obtained from cBioPortal (14).

Prediction of the regulatory miRNAs of $L B X 2$. The regulatory miRNAs of LBX2 were predicted using four miRNAs target prediction databases: TargetScan version 7.2 (targetscan. org/vert_72), miRDB (mirdb.org/), miRWalk (129.206.7.150) and microRNA (microrna.org/microrna/home.do). The common miRNAs in at least three of the target prediction databases were defined as the prediction cohort using Venny version 2.1.0 (bioinfogp.cnb.csic.es/tools/venny/index.html). Compared with normal controls, the miRNAs downregulated in TCGA colon adenocarcinoma and rectum adenocarcinoma cohorts (also present in the prediction cohort) were regarded as the candidate regulatory miRNAs of LBX2 in CRC. In addition, linear regression analyses were performed to assess the association between LBX2 mRNA expression levels and
miRNAs expression. Kaplan-Meier analyses were performed to determine the prognostic value of candidate regulatory miRNAs of LBX2 in CRC.

Gene set enrichment analysis (GSEA). GSEA software version 3.0 was used to evaluate the correlations between LBX2 expressions (high vs. low) and tumor-associated pathways in the TCGA colon adenocarcinoma and rectum adenocarcinoma datasets. The reference gene set C2 (c2.cp.kegg.v6.0.symbols. gmt), which summarizes and represents specific, well-defined, biological signal pathways and processes, belongs to the Molecular Signatures Database (software.broadinstitute. org/gsea/msigdb/index.jsp) (15). The normalized enrichment score (NES) was obtained by analyzing with permutations for 1,000 times. A gene set was deemed to be significantly enriched when the normal P-value $<0.05$ and the false discovery rate $(\mathrm{FDR})<0.25$.

Statistical analysis. A two-tailed Student's t-test was used to perform expression data comparisons in different clinicopathological groups using the GraphPad Prism 7 software (GraphPad Software). One-way ANOVA and Tukey's post-hoc test were used for the comparison of datasets containing three or more groups. Receiver operating characteristic (ROC) curves were established to estimate the diagnostic value of LBX2 in CRC. Patients were then divided into low and high LBX2 expression groups based on survival data using the X-tile software version 3.6.1 (16), which automatically chooses the optimum cut-off value. The correlations between LBX2 expression levels and clinicopathological parameters were evaluated using SPSS software (version 22.0; IBM Corp.) with the application of the two-tailed $\chi^{2}$ test. Overall survival (OS) and disease-free survival (DFS) were determined using Kaplan-Meier analysis and a log-rank test. SPSS was employed to perform univariate and multivariable analyses to generate a Cox proportional hazards model for the analysis of relative risk factors associated with OS or DFS. The Pearson's correlation analysis was used to determine the linear relationship between the LBX2 mRNA expression levels and the LBX2 DNA methylation level or the miRNAs expression. $\mathrm{P}<0.05$ was considered to indicate a statistically significant difference.

\section{Results}

LBX2 is overexpressed in CRC tissues compared with normal colorectal tissues. The Oncomine database was used to analyze LBX2 mRNA expression levels in colorectal tumors and corresponding normal tissues. As shown in Fig. 1A, compared with normal tissues, LBX2 expression levels were higher in CRC. In addition, a lower LBX2 expression was observed in cervical and gastric cancer.

To assess LBX2 expression in the various types of cancer further, the LBX2 expression levels in multiple malignant tumors in TCGA were analyzed using mRNA expression data from TIMER. The difference in LBX2 expression levels between tumor and adjacent normal tissues across all TCGA tumors are shown in Fig. 1B. LBX2 expression levels were significantly higher in bladder urothelial carcinoma, breast invasive carcinoma, colon adenocarcinoma, esophageal carcinoma, head and neck cancer, kidney renal clear cell carcinoma, 

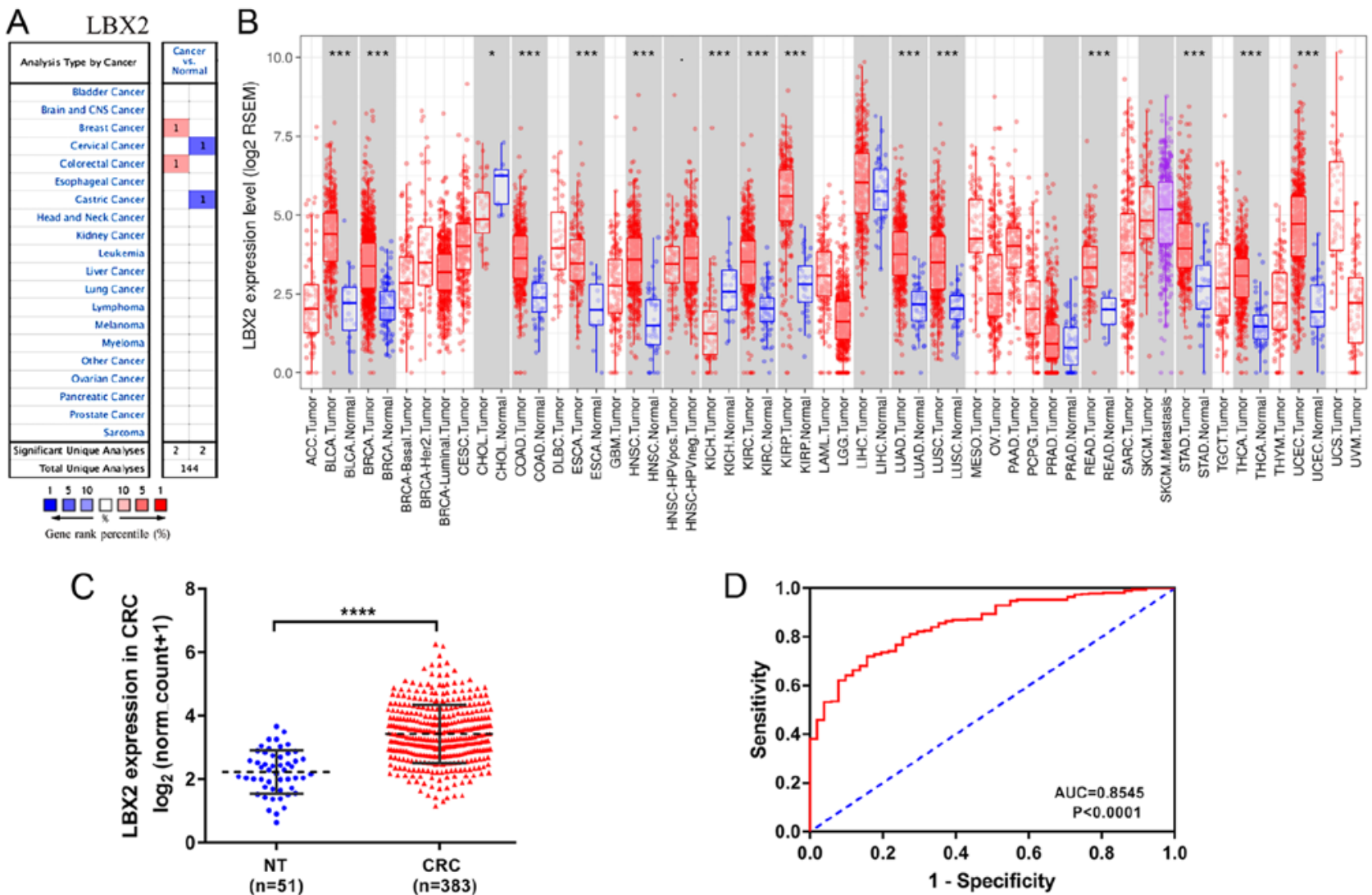

Figure 1. LBX2 mRNA expression profiles in different types of cancer. (A) Increased or decreased LBX2 in data sets of different types of cancer compared with normal tissues in the Oncomine database. (B) LBX2 expression levels in different tumor types from the Cancer Genome Atlas database were examined using the Tumor Immune Estimation Resource. (C) Comparison of LBX2 expression levels in CRC tissues ( $\mathrm{n}=383$ ) and in normal colorectal tissues $(\mathrm{n}=51)$. (D) Receiver Operating Characteristic curves were used to validate LBX2 overexpression for CRC survival prediction. ${ }^{*} \mathrm{P}<0.05,{ }^{* *} \mathrm{P}<0.01,{ }^{* * *} \mathrm{P}<0.001$, ${ }^{* * * * *} \mathrm{P}<0.0001$. NT, normal tissue; CRC, colorectal cancer; AUC, area under the curve; ACC, adrenocortical carcinoma; BLCA, bladder urothelial carcinoma; BRCA, breast invasive carcinoma; CESC, cervical squamous cell carcinoma and endocervical adenocarcinoma; CHOL, cholangiocarcinoma; COAD, colon adenocarcinoma; DLBC, lymphoid neoplasm diffuse large B-cell lymphoma; ESCA, esophageal carcinoma; GBM, glioblastoma multiforme; HNSC, head and neck squamous cell carcinoma; KICH, kidney chromophobe; KIRC, kidney renal clear cell carcinoma; KIRP, kidney renal papillary cell carcinoma; LAML, acute myeloid leukemia; LGG, brain lower grade glioma; LIHC, liver hepatocellular carcinoma; LUAD, lung adenocarcinoma; LUSC, lung squamous cell carcinoma; MESO, mesothelioma; OV, ovarian serous cystadenocarcinoma; PAAD, pancreatic adenocarcinoma; PCPG, pheochromocytoma and paraganglioma; PRAD, prostate adenocarcinoma; READ, rectum adenocarcinoma; SARC, sarcoma; SKCM, skin cutaneous melanoma; STAD, stomach adenocarcinoma; TGCT, testicular germ cell tumors; THCA, thyroid carcinoma; THYM, thymoma; UCEC, uterine corpus endometrial carcinoma; UCS, uterine carcinosarcoma; UVM, uveal melanoma.

lung adenocarcinoma, lung squamous cell carcinoma, rectum adenocarcinoma, stomach adenocarcinoma, thyroid carcinoma and uterine corpus endometrial carcinoma, compared with normal tissues. Conversely, LBX2 expression levels were significantly lower in cholangiocarcinoma and kidney chromophobe compared with adjacent normal tissues.

Furthermore, the quantitative evaluation of the expression levels of LBX2 mRNA in CRC samples ( $n=383$ ) showed that LBX2 mRNA expression levels were significantly higher compared with normal colorectal tissues $(n=51$; $\mathrm{P}<0.0001$; Fig. 1C). The AUC value of LBX2 overexpression for CRC diagnosis was $0.8545(\mathrm{P}<0.0001)$ (Fig. 1D). These results suggest that $\mathrm{LBX} 2$ is upregulated in multiple types of cancer, particularly in CRC.

Association between LBX2 expression levels and survival of patients with CRC. Overall, 383 patients with CRC with complete LBX2 mRNA sequencing data and clinical data in TCGA colon adenocarcinoma and rectum adenocarcinoma datasets were analyzed to explore the clinical significance of
LBX2. As shown in Table I, the expression levels of LBX2 were not associated with patients' age, perineural infiltration and residual tumor (all $\mathrm{P}>0.05$ ), whereas significant correlations were observed between high LBX2 expression levels and male patients, advanced tumor stage (17) (III or IV), vascular invasion, lymphatic invasion, recurrence or progression and living status (all $\mathrm{P}<0.05$ ). Consistent with these findings, $\mathrm{LBX} 2$ mRNA expression levels data were analyzed as a continuous variable, which further demonstrated the different LBX2 expression levels among the different clinicopathological groups (sex, tumor stage, lymphatic or vascular invasion, disease-free status and living status) (all $\mathrm{P}<0.05$; Fig. 2).

LBX2 overexpression independently predicts poor OS and $D F S$ in CRC. To explore the prognostic value of LBX2 expression levels, the Kaplan-Meier method was used to analyze the effects of LBX2 expression on the OS and DFS of patients with CRC. The results showed that high LBX2 expression levels were significantly correlated with less favorable OS and DFS (all P<0.05; Fig. 3). 
Table I. Association between LBX2 expression levels and clinical characteristics in patients with colorectal cancer in The Cancer Genome Atlas.

\begin{tabular}{|c|c|c|c|c|}
\hline \multirow[b]{2}{*}{ Characteristic } & \multicolumn{2}{|c|}{ LBX2 expression } & \multirow[b]{2}{*}{$\chi^{2}$ value } & \multirow[b]{2}{*}{ P-value } \\
\hline & Low, n=191, (\%) & High, n=192, (\%) & & \\
\hline Age, years & & & 0.3137 & 0.5754 \\
\hline$<65$ & 93 (48.7) & $88(45.8)$ & & \\
\hline$\geq 65$ & $98(51.3)$ & $104(54.2)$ & & \\
\hline Sex & & & 6.841 & $0.0089^{b}$ \\
\hline Male & 93 (48.7) & $119(62.0)$ & & \\
\hline Female & $98(51.3)$ & $73(38.0)$ & & \\
\hline Tumor stage & & & 6.758 & $0.0341^{\mathrm{a}}$ \\
\hline I-II & $108(56.6)$ & $88(45.9)$ & & \\
\hline III-IV & $73(38.2)$ & $93(48.4)$ & & \\
\hline Unknown & $10(5.2)$ & $11(5.7)$ & & \\
\hline Vascular invasion & & & 6.519 & $0.0384^{\mathrm{a}}$ \\
\hline No & $138(72.3)$ & $115(59.9)$ & & \\
\hline Yes & $31(16.2)$ & 45 (23.4) & & \\
\hline Unknown & $22(11.5)$ & $32(16.7)$ & & \\
\hline Lymphatic invasion & & & 8.539 & $0.0140^{\mathrm{a}}$ \\
\hline No & $125(65.5)$ & $105(54.7)$ & & \\
\hline Yes & $44(23.0)$ & $58(30.2)$ & & \\
\hline Unknown & $22(11.5)$ & $29(15.1)$ & & \\
\hline Paraneural infiltration & & & 0.7739 & 0.6791 \\
\hline No & $83(43.5)$ & $86(44.8)$ & & \\
\hline Yes & $32(16.7)$ & $26(13.5)$ & & \\
\hline Unknown & $76(39.8)$ & $80(41.7)$ & & \\
\hline Residual tumor & & & 2.207 & 0.3318 \\
\hline R0 & $121(63.3)$ & $126(65.6)$ & & \\
\hline $\mathrm{R} 1-\mathrm{R} 2$ & $3(1.6)$ & $7(3.7)$ & & \\
\hline RX-unknown & $67(35.1)$ & $59(30.7)$ & & \\
\hline Disease-free status & & & 9.209 & $0.0100^{\mathrm{b}}$ \\
\hline Disease-free & $134(70.1)$ & $106(55.2)$ & & \\
\hline Recurrence & $37(19.4)$ & $54(28.1)$ & & \\
\hline Unknown & $20(10.5)$ & $32(16.7)$ & & \\
\hline Living status & & & 11.99 & $0.0025^{\mathrm{b}}$ \\
\hline Alive & 157 (82.2) & $134(69.8)$ & & \\
\hline Dead & $30(15.7)$ & $57(29.7)$ & & \\
\hline Unknown & $4(2.1)$ & $1(0.5)$ & & \\
\hline
\end{tabular}

${ }^{\mathrm{a}} \mathrm{P}<0.05 ;{ }^{\mathrm{b}} \mathrm{P}<0.01$.

Furthermore, the independent risk factors of OS and DFS were evaluated using the Cox proportional hazards regression model. Univariate and multivariate analyses demonstrated that OS was significantly associated with LBX2 expression levels, age and tumor stage, and vascular and lymphatic invasion (only univariate analysis) (all $\mathrm{P}<0.05$; Table II). Conversely, univariate and multivariate analyses demonstrated that DFS was significantly associated with LBX2 expression levels and tumor stage, and vascular and lymphatic invasion and residual tumor (only univariate analysis) (all $\mathrm{P}<0.05$; Table III). Multivariate Cox regression analyses further demonstrated that upregulation of LBX2 was an independent risk factor of a less favorable OS [high vs. low; hazard ratio (HR), 2.934; confidence interval (CI), 1.735-4.965; $\mathrm{P}<0.001]$ and DFS (high vs. low; HR, 2.135; CI, 1.183-3.853; P=0.012) (Tables II and III).

DNA hypomethylation and microRNA (miR)-378a-3p dysregulation contribute to LBX2 upregulation in CRC. To further explore the potential mechanisms of LBX2 upregulation in CRC, the genetic and epigenetic alterations of LBX2 in TCGA colon adenocarcinoma and rectum adenocarcinoma datasets were analyzed. According to the tumor samples with complete 

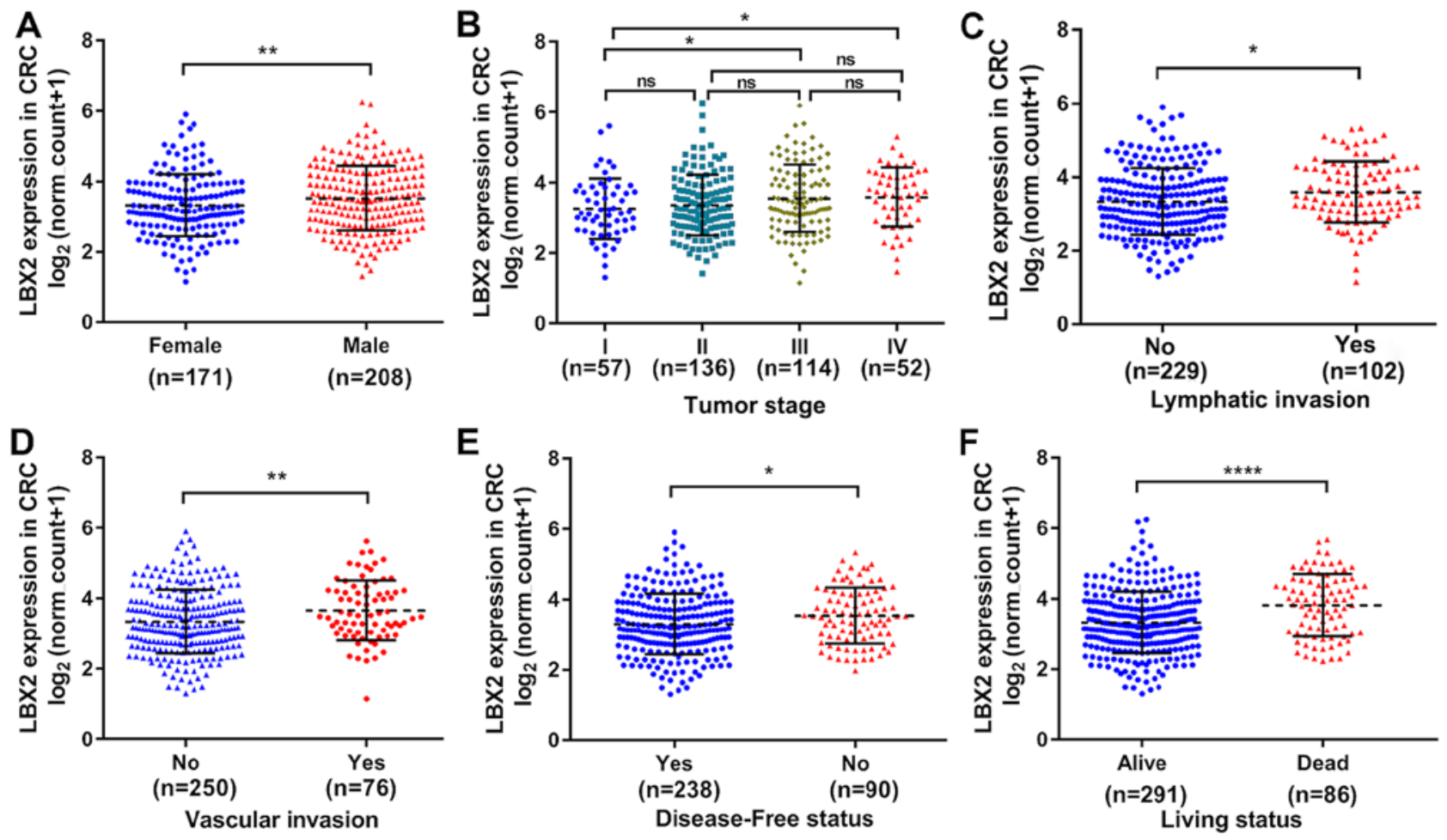

Figure 2. Comparison of LBX2 expression levels in different clinicopathological groups. Comparison of LBX2 expression in different groups of: (A) sex; (B) tumor stage; (C) lymphatic invasion; (D) vascular invasion, (E) disease-free status and (F) living status. ${ }^{*} \mathrm{P}<0.05,{ }^{* *} \mathrm{P}<0.01,{ }^{* * * * *} \mathrm{P}<0.0001 . \mathrm{ns}$, not significant.
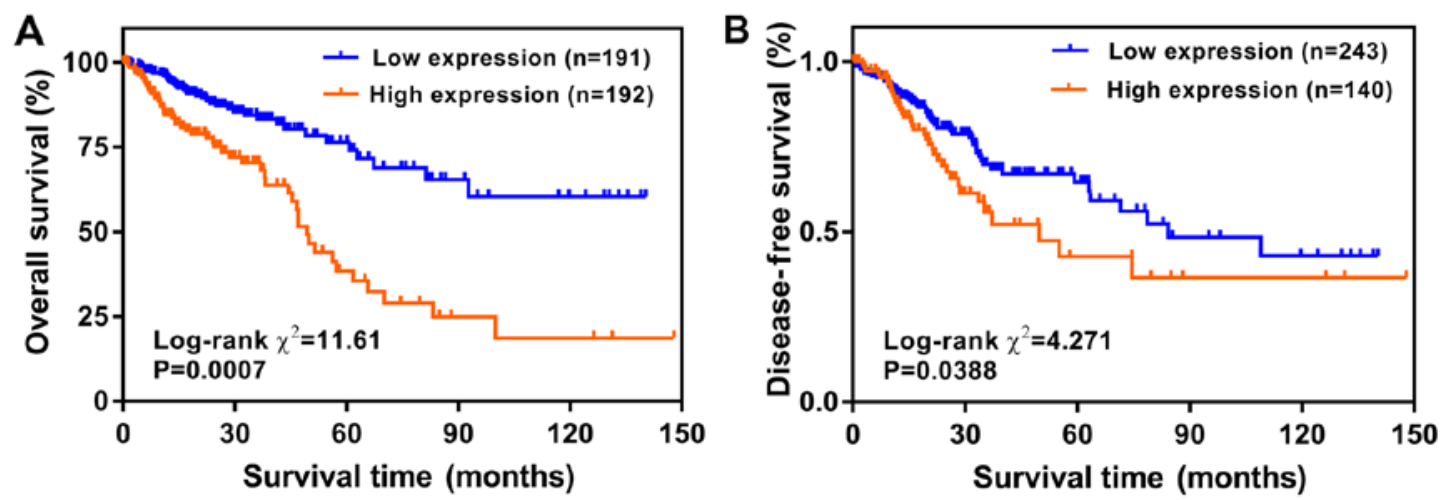

Figure 3. Kaplan-Meier survival curves of 383 cases of CRC according to LBX2 expression levels. (A) Over survival curve of patients with CRC based on LBX2 expression levels. (B) Disease-free survival curve of patients with CRC based on LBX2 expression levels. CRC, colorectal cancer.

mRNA, CNA and methylation data $(\mathrm{n}=372), 4$ patients with CRC harbored a LBX2 shallow deletion (single copy deletion) and 88 patients harbored a LBX2 DNA copy gain (low-level amplification); however the LBX2 CNA was not significantly correlated with the upregulation of LBX2 mRNA ( $>>0.05$; Fig. 4A). The DNA methylation levels of LBX2 between normal colorectal tissues and CRC samples were compared and no significant difference was observed ( $\mathrm{P}>0.05$; Fig. 4B). The association between LBX2 mRNA expression levels and LBX2 DNA methylation was also analyzed. Linear regression analyses indicated that the DNA methylation levels of LBX2 were correlated moderately and negatively with LBX2 mRNA expression levels (Pearson's $r=-0.2905$; $\mathrm{P}<0.001$; Fig. 4C). These results suggest that the DNA methylation of LBX2 may not directly participate in carcinogenesis. However, the upregulation of LBX2 in CRC may be associated with the hypermethylation of LBX2 and this requires further investigation.

Additionally, the present study sought to identify the possible regulatory miRNAs of LBX2. Candidate regulatory miRNAs $(n=22)$ of LBX2 were obtained from four miRNAs target prediction platforms (Fig. 5A). Moreover, data from TCGA database showed that two miRNAs (miRs), miR-378a-3p and miR-339-5p, of the 22 candidate regulatory miRNAs were significantly downregulated in CRC tissues compared with normal colorectal tissues and a possible miR-378a-3p/LBX2 alignment was acquired from miRNAs target prediction databases (Fig. 5B-D). Considered as candidates for regulatory miRNAs of LBX2, miR-378a-3p and miR-339-5p were used for further analyses and validation. Linear regression analyses revealed that miR-378a-3p expression levels were correlated moderately and negatively with LBX2 mRNA expression levels 
Table II. Univariate and multivariate Cox regression analyses of overall survival for patients with colorectal cancer.

\begin{tabular}{|c|c|c|c|c|}
\hline \multirow[b]{2}{*}{ Variables } & \multicolumn{2}{|c|}{ Univariate analysis } & \multicolumn{2}{|c|}{ Multivariate analysis } \\
\hline & $\mathrm{HR}(95 \% \mathrm{CI})$ & P-value & $\mathrm{HR}(95 \% \mathrm{CI})$ & P-value \\
\hline LBX2, high vs. low & $2.791(1.816-4.289)$ & $<0.001^{\mathrm{b}}$ & $2.934(1.735-4.965)$ & $<0.001^{\mathrm{b}}$ \\
\hline Age, years, $\geq 65$ vs. $<65$ & $1.997(1.261-3.161)$ & $0.008^{\mathrm{a}}$ & $2.247(1.299-3.887)$ & $0.004^{\mathrm{a}}$ \\
\hline Sex, female vs. male & $1.219(0.795-1.870)$ & 0.363 & - & - \\
\hline Tumor stage, III-IV vs. I-II & $2.943(1.829-4.735)$ & $<0.001^{\mathrm{b}}$ & $3.349(1.875-5.983)$ & $<0.001^{\mathrm{b}}$ \\
\hline Vascular invasion, yes vs. no & $2.446(1.521-3.934)$ & $<0.001^{\mathrm{b}}$ & $2.223(1.137-4.346)$ & 0.156 \\
\hline Paraneural infiltration, yes vs. no & $1.552(0.819-2.938)$ & 0.178 & - & - \\
\hline Lymphatic invasion, yes vs. no & $1.703(1.084-2.675)$ & $0.008^{\mathrm{a}}$ & $0.653(0.327-1.304)$ & 0.325 \\
\hline Residual tumor, R1-R2 vs. R0 & $1.920(0.675-5.460)$ & 0.222 & - & - \\
\hline
\end{tabular}

${ }^{\mathrm{a}} \mathrm{P}<0.01 ;{ }^{\mathrm{b}} \mathrm{P}<0.001$. HR, hazard ratio; $\mathrm{CI}$, confidence interval.

Table III. Univariate and multivariate Cox regression analyses of disease-free survival for patients with colorectal cancer.

\begin{tabular}{|c|c|c|c|c|}
\hline \multirow[b]{2}{*}{ Variables } & \multicolumn{2}{|c|}{ Univariate analysis } & \multicolumn{2}{|c|}{ Multivariate analysis } \\
\hline & HR $(95 \% \mathrm{CI})$ & P-value & HR $(95 \% \mathrm{CI})$ & P-value \\
\hline LBX2, high vs. low & $1.685(1.112-2.555)$ & $0.014^{\mathrm{a}}$ & $2.135(1.183-3.853)$ & $0.012^{\mathrm{a}}$ \\
\hline Age, years, $\geq 65$ vs. $<65$ & $0.900(0.596-1.358)$ & 0.616 & - & - \\
\hline Sex, female vs. male & $1.387(0.906-2.213)$ & 0.132 & - & - \\
\hline Tumor stage, III-IV vs. I-II & $2.599(1.705-3.906)$ & $<0.001^{\mathrm{b}}$ & $1.952(1.016-3.750)$ & $0.045^{\mathrm{a}}$ \\
\hline Vascular invasion, yes vs. no & $1.762(1.109-2.801)$ & $0.017^{\mathrm{a}}$ & $1.187(0.530-2.658)$ & 0.677 \\
\hline Paraneural infiltration, yes vs. no & $1.396(0.800-2.437)$ & 0.240 & - & - \\
\hline Lymphatic invasion, yes vs. no & $2.291(1.501-3.495)$ & $<0.001^{\mathrm{b}}$ & $1.630(0.508-2.664)$ & 0.721 \\
\hline Residual tumor, R1-R2 vs. R0 & $2.530(1.074-5.962)$ & $0.034^{\mathrm{a}}$ & $2.384(0.951-5.977)$ & 0.064 \\
\hline
\end{tabular}

${ }^{\mathrm{a}} \mathrm{P}<0.05 ;{ }^{\mathrm{b}} \mathrm{P}<0.001$. HR, hazard ratio; $\mathrm{CI}$, confidence interval.
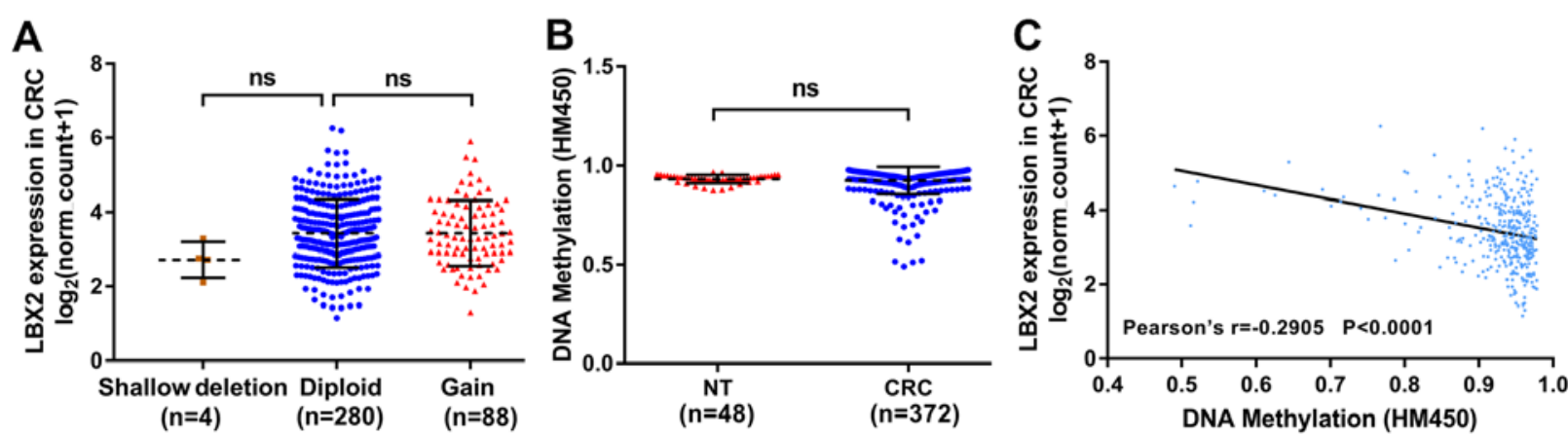

Figure 4. Association between LBX2 upregulation and DNA copy number alterations or hypomethylation in patients with CRC. (A) LBX2 mRNA expression levels in CRC tissues with indicating DNA copy number alterations. (B) Comparison of LBX2 DNA methylation between CRC samples and normal colorectal tissues. (C) Correlation between LBX2 mRNA expression levels and LBX2 DNA methylation. ns, not significant; NT, normal tissue; CRC, colorectal cancer.

(Pearson's r=-0.264; $\mathrm{P}<0.001$; Fig. 5E), whereas miR-339-5p expression levels were not significantly correlated with LBX2 mRNA expression levels (Pearson's $\mathrm{r}=-0.0128 ; \mathrm{P}=0.8312$; Fig. 5E). Kaplan-Meier analyses further demonstrated that low miR-378a-3p expression levels were linked to less favorable OS and DFS (all $\mathrm{P}<0.05$; Fig. $6 \mathrm{~A}$ and $\mathrm{B}$ ). However, no significance was observed between miR-339-5p expression levels and OS or DFS (all P>0.05; Fig. 6C and D). These results suggest that miR-378a-3p could serve as a potential regulator of LBX2 in $\mathrm{CRC}$ and needs to be researched further.

Gene set enrichment analysis. GSEA was performed to investigate the underlying biological functions of LBX2 upregulation in CRC. Four gene sets, 'VEGF_SIGNALING_PATHWAY', 

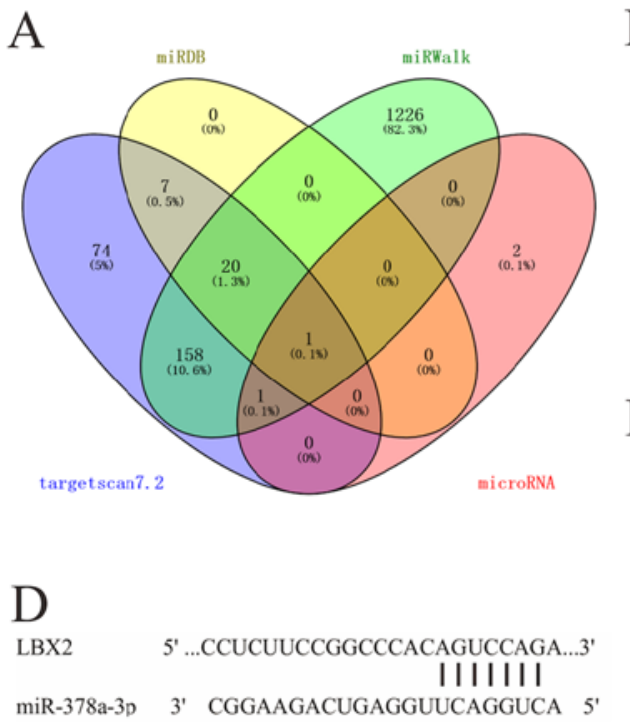
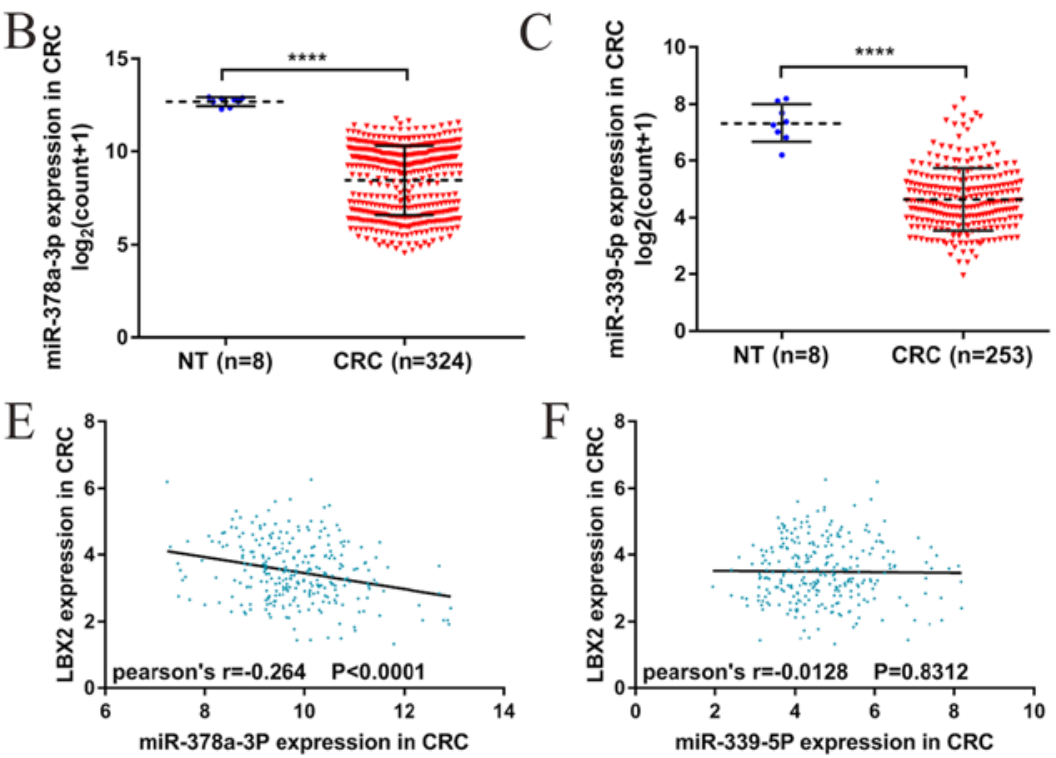

Figure 5. Downregulation of miR-378a-3p contributes to LBX2 upregulation in CRC. (A) Selection of potential regulatory miRNAs of LBX2 in miRNAs target prediction platforms. (B) Comparison of miR-378a-3p expression levels in CRC tissues to normal colorectal tissues. (C) Comparison of miR-339-5p expression levels in CRC tissues to normal colorectal tissues. (D) The possible binding site of LBX2 3'UTR by miR-378a-3p. (E) Correlation between the miR-378a-3p expression levels and LBX2 mRNA expression levels. (F) Correlation between the miR-339-5p expression levels and LBX2 mRNA expression levels. ${ }^{* * * *} \mathrm{P}<0.0001$. miR, microRNA; CRC, colorectal cancer; UTR, untranslated region; NT, normal tissue.
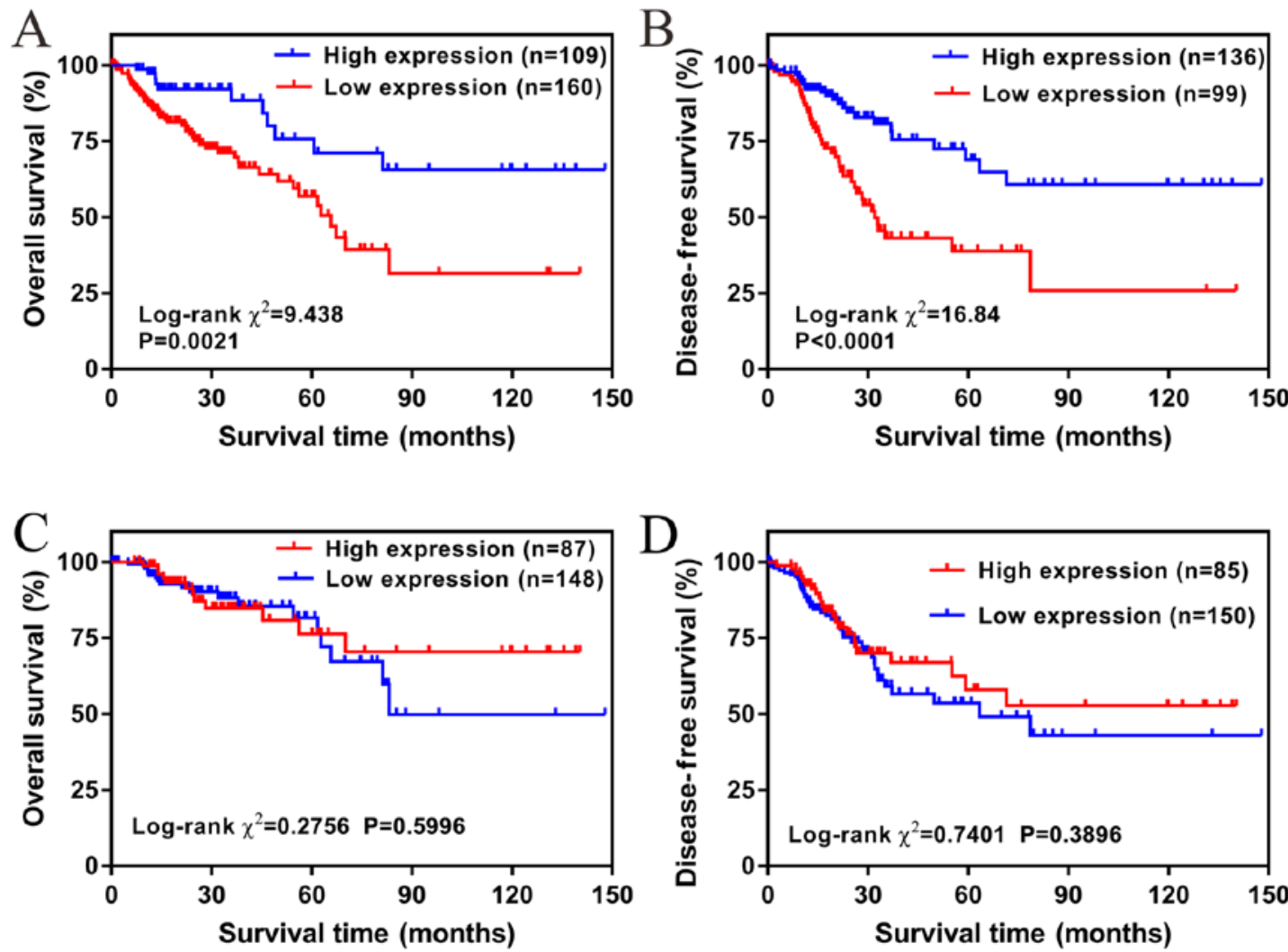

Figure 6. Kaplan-Meier survival curves comparing the high and low expression of miR-378a-3p in CRC. Low expression of miR-378a-3p in patients with CRC is associated with less favorable (A) OS and (B) DFS. Expression levels of miR-339-5p in patients with CRC is not significantly correlated with (C) OS and (D) DFS. miR, microRNA; CRC, colorectal cancer; OS, overall survival; DFS, disease free survival.

'CELL_ADHESION_MOLECULES_CAMS', 'TOLL_LIKE RECEPTOR_SIGNALING_PATHWAY', 'NATURAL_ LILLER_CELL_MEDIATED_CYTOTOXICITY', were significantly enriched (all $\mathrm{P}<0.05$; Fig. 7).

\section{Discussion}

In 2018, CRC was reported as the third most commonly diagnosed type of cancer and the leading cause of cancer-associated 

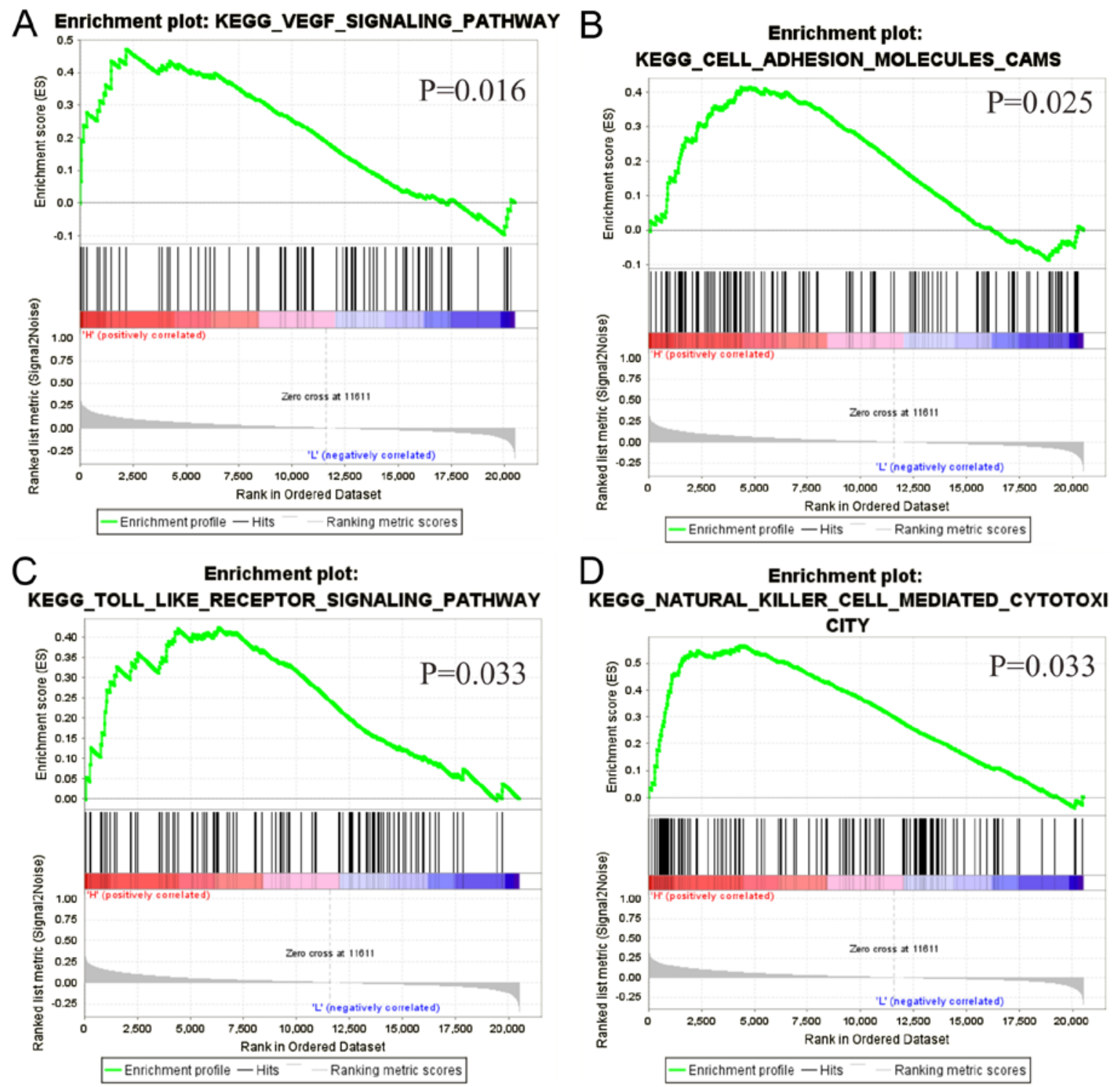

Figure 7. Gene set enrichment analysis (GSEA) of LBX2 upregulation in colorectal cancer. Upregulation of LBX2 was significantly associated with (A) 'VEGF signaling', (B) 'Cell adhesion molecules CAMs', (C) 'Toll-like receptor signaling' and (D) 'Natural killer cell mediated cytotoxicity' pathways. KEGG, Kyoto Encyclopedia of Genes and Genomes.

morality worldwide (2). Efforts have been made in establishing the early detection and comprehensive therapy of CRC (18). Nevertheless, individual prognosis prediction remains challenging due to the high heterogeneity and complex molecular biological characteristics of CRC (18). Several investigations on tumor biology from the past few years have provided insights on how to improve the effectiveness of treatments through patient stratification, which has improved the prognosis of patients with CRC $(18,19)$. Furthermore, the availability of comprehensive genetic and molecular profiling has created promising treatment opportunities for CRC (19). Hence, it is essential to investigate the biological characteristics of CRC further, as well as develop new biomarkers for the stratification of patients with CRC into different prognostic subgroups to improve treatment decision-making for CRC.

LBX2 is located on chromosome 2p13.1 and several previous studies have reported that it is dysregulated in non-small cell lung cancer, adenoid cystic carcinoma and T-cell acute lymphoblastic leukemia (11-13). However, the expression levels of LBX2 and its clinical significance in CRC has not been investigated before, to the best of our knowledge. In the present study, it was initially demonstrated that LBX2 at the mRNA level was significantly upregulated in CRC tissues compared with normal colorectal tissues. Based on ROC curves, the diagnostic value of LBX2 mRNA expression reached high efficiency, indicating that LBX2 may be a promising biomarker for the diagnosis of CRC.

Investigation into the clinical significance of LBX2 expression levels in CRC tissues revealed that the overexpression of LBX2 was associated with male patients, advanced tumor stage, and vascular and lymphatic invasions, suggesting that LBX2 may be involved in the occurrence and progression of CRC. Kaplan-Meier analyses showed that LBX2 upregulation was correlated with less favorable OS and DFS. Multivariate analyses further confirmed that LBX2 upregulation was an independent risk factor of less favorable OS in patients with CRC. These results suggest that the expression levels of LBX2 may be a valuable prognostic indicator and promising monitoring 
indicator of postoperative tumor recurrence in patients with CRC. Approximately 10-35\% of patients with CRC suffer a recurrence within 5 years of diagnosis despite achieving remission after curative resection and/or chemotherapy (20-22).

Serum carcinoembryonic antigen (CEA) monitoring is one of the recommended indicators for early detection of disease recurrence or second primary cancer (23). Although CEA is the most widely used CRC molecular marker, CEA can also be elevated in other types of cancer, such as ovarian, pancreatic, gastric, lung and breast cancer and benign conditions, including inflammatory bowel disease and pancreatitis (23). Thus, identifying another clinical biomarker to use independently or combined with CEA in CRC monitoring for recurrence and metastasis is important. The analyses of the present study showed that LBX2 was significantly upregulated in CRC tissues compared with normal colorectal tissues, but the difference in the expression level of serum LBX2 between patients with CRC and healthy individuals remains unclear. Therefore, the diagnostic and prognostic values of independent serum LBX2 or combined with CEA in CRC requires further study.

The underlying mechanisms of LBX2 upregulation in CRC were further explored. LBX2 is located on chromosome 2 p13.1, which has been identified differentially expressed in several malignancies (11-13). From the perspective of genetic and epigenetic alterations, it was demonstrated that 88 patients with CRC had low-level LBX2 DNA amplification, but this was not significantly correlated with LBX2 expression levels. In addition, DNA methylation is an essential regulator of gene transcription and hypermethylation represses the transcription of the promoter regions of tumor suppressor genes leading to gene silencing, while hypomethylation is considered as a cause of oncogenesis (24). Consistent with this knowledge, the present study demonstrated that LBX2 DNA hypomethylation was significantly correlated with the upregulation of LBX2 expression levels.

Furthermore, miRNAs are critical regulators of gene expression that can repress gene expression by binding to the 3 ' untranslated region (3'UTR) of mRNAs to target them for degradation and thereby prevent their translation (25). Considering the deregulation of miRNA functioning involved in several types of human cancer (26), the present study aimed to identify the candidate regulatory miRNAs of LBX2 and demonstrated that miR-378-3p and miR-339-5p were significantly downregulated in CRC samples compared with normal colorectal tissues. Additional linear regression analyses showed that miR-378-3p expression, but not that of miR-339-5p, were negatively correlated with LBX2 mRNA expression levels. Kaplan-Meier analyses also revealed that miR-378-3p downregulation, but not that of miR-339-5p, was associated with a less favorable OS and DFS. Several previous studies showed that miR-378-3p was downregulated in $\mathrm{CRC}$, esophageal squamous cell carcinoma and prostate cancer (27-29). In addition, miR-378-3p has been confirmed to be downregulated in CRC compared with adjacent normal colorectal tissues (28-30), consistent with the present study, and could serve as an independent prognostic marker or potential target of novel cancer therapies. The putative binding of miR-378-3p at the 3'UTR site of LBX2 further supports the hypothesis that miR-378-3p may be an upstream regulator of LBX2 in CRC. These results indicate that both LBX2 DNA hypomethylation and $\mathrm{miR}-378-3 \mathrm{p}$ downregulation contribute to LBX2 dysregulation in CRC.

The potential biological pathways of LBX2 upregulation in CRC remain to be elucidated further. The findings of the present study from GSEA suggested that high LBX2 expression levels in CRC were correlated with 'VEGF signaling', 'Cell adhesion molecules CAMs', 'Toll-like receptor signaling' and 'Natural killer cell-mediated cytotoxicity' which have been showed to be involved in CRC development and progression (31-34). Furthermore, the present study showed that the high expression levels of LBX2 were significantly associated with advanced tumor stage of CRC. To the best of our knowledge, no current studies has investigated the association between LBX2 and CRC. Hence, with little research on this subject, the underlying regulatory mechanisms of LBX2 in $\mathrm{CRC}$ require further research.

Although the present study investigated several independent databases, there are some limitations. First, the clinical significance of LBX2 in CRC was only investigated using patient samples from TCGA database and lack validation using further clinical samples. In addition, experiments investigating the association between LBX2 expression levels and the invasion and migration of CRC were not performed in the present study. Further studies are needed to understand the underlying mechanisms of LBX2 function in CRC.

In summary, LBX2 expression levels were significantly upregulated in CRC tissues compared with normal colorectal tissues, which might have been caused by LBX2 DNA hypomethylation and miR-378a-3p downregulation in CRC. Importantly, the present research indicates that LBX2 overexpression was associated with tumor progression and was an independent predictor of less favorable OS and DFS in patients with CRC. The addition of LBX2 assessment to prognosis may lead to more accurate survival stratification of patients with CRC and may enable more appropriate clinical treatment decision-making. Furthermore, LBX2 overexpression was significantly associated with 'VEGF signaling', 'Cell adhesion molecules CAMs', 'Toll-like receptor signaling' and 'Natural killer cell-mediated cytotoxicity' pathways in CRC. Additional studies are needed to clarify the underlying mechanisms of LBX2 in CRC.

\section{Acknowledgements}

Not applicable.

\section{Funding}

No funding was received.

\section{Availability of data and materials}

The datasets generated and/or analyzed during the present study are available in The Cancer Genome Atlas repository (cancer.gov/tcga).

\section{Authors' contributions}

$\mathrm{XH}$ and $\mathrm{YZ}$ conceived and designed the present study, performed statistical analysis and drafted the initial manuscript. 
YY, CY, HL and HC made substantial contributions to data analysis and manuscript modifications. All authors read and approved the final manuscript.

\section{Ethics approval and consent to participate}

Not applicable.

\section{Patient consent for publication}

Not applicable.

\section{Competing interests}

The authors declare that they have no competing interests.

\section{References}

1. Bray F, Ferlay J, Soerjomataram I, Siegel RL, Torre LA and Jemal A: Global cancer statistics 2018: GLOBOCAN estimates of incidence and mortality worldwide for 36 cancers in 185 countries. CA Cancer J Clin 68: 394-424, 2018.

2. Siegel RL, Miller KD, Fedewa SA, Ahnen DJ, Meester RG, Barzi A and Jemal A: Colorectal cancer statistics, 2017. CA Cancer J Clin 67: 177-193, 2017.

3. Cancer Genome Atlas Network: Comprehensive molecular characterization of human colon and rectal cancer. Nature 487: 330-337, 2012.

4. Mogensen MB, Rossing M, Østrup O, Larsen PN, Heiberg Engel PJ, Jørgensen LN, Hogdall EV, Eriksen J, Ibsen P, Jess $\mathrm{P}$, et al: Genomic alterations accompanying tumour evolution in colorectal cancer: Tracking the differences between primary tumours and synchronous liver metastases by whole-exome sequencing. BMC Cancer 18: 752, 2018.

5. Wang $X$, Fang $H$, Cheng Y, Li L, Sun X, Fu T, Huang P, Zhang A, Feng Z, Li C, et al: The molecular landscape of synchronous colorectal cancer reveals genetic heterogeneity. Carcinogenesis 39: 708-718, 2018.

6. Grasso CS, Giannakis M, Wells DK, Hamada T, Mu XJ, Quist M, Nowak JA, Nishihara R, Qian ZR, Inamura K, et al: Genetic mechanisms of immune evasion in colorectal cancer. Cancer Discov 8: 730-749, 2018.

7. Wang J, Luo J, Chen Q, Wang X, He J, Zhang W, Yin Z, Zheng F, $\mathrm{Pan} \mathrm{H}, \mathrm{Li}$, et al: Identification of LBX2 as a novel causal gene of atrial septal defect. Int J Cardiol 265: 188-194, 2018.

8. Lou Q, He J, Hu L and Yin Z: Role of lbx2 in the noncanonical Wnt signaling pathway for convergence and extension movements and hypaxial myogenesis in zebrafish. Biochim Biophys Acta 1823: 1024-1032, 2012.

9. Lu FI, Sun YH, Wei CY, Thisse C and Thisse B: Tissue-specific derepression of TCF/LEF controls the activity of the Wnt/ $\beta$-catenin pathway. Nat Commun 5: 5368, 2014.

10. Ochi $\mathrm{H}$ and Westerfield $\mathrm{M}$ : Lbx2 regulates formation of myofibrils. BMC Dev Biol 9: 13, 2009.

11. Wang J, Song J, Gao Z, Huo X, Zhang Y, Wang W, Qi J and Zheng S: Analysis of gene expression profiles of non-small cell lung cancer at different stages reveals significantly altered biological functions and candidate genes. Oncol Rep 37: 1736-1746, 2017.

12. Bell A, Bell D, Weber RS and El-Naggar AK: CpG island methylation profiling in human salivary gland adenoid cystic carcinoma. Cancer 117: 2898-2909, 2011.

13. Villarese P, Lours C, Trinquand A, Le Noir S, Belhocine M, Lhermitte L, Cieslak A, Tesio M, Petit A, LeLorch M, et al: TCR $\alpha$ rearrangements identify a subgroup of NKL-deregulated adult T-ALLs associated with favorable outcome. Leukemia 32: 61-71, 2018.

14. Cerami E, Gao J, Dogrusoz U, Gross BE, Sumer SO, Aksoy BA, Jacobsen A, Byrne CJ, Heuer ML, Larsson E, et al: The cBio cancer genomics portal: An open platform for exploring multidimensional cancer genomics data. Cancer Discov 2: 401-404, 2012.
15. Subramanian A, Tamayo P, Mootha VK, Mukherjee $S$, Ebert BL, Gillette MA, Paulovich A, Pomeroy SL, Golub TR, LanderES, et al:Gene setenrichment analysis: A knowledge-based approach for interpreting genome-wide expression profiles. Proc Natl Acad Sci USA 102: 15545-15550, 2005.

16. Camp RL, Dolled-Filhart M and Rimm DL: X-tile: a new bio-informatics tool for biomarker assessment and outcome-based cut-point optimization. Clinical cancer research : an official journal of the American Association for Cancer Research 10: 7252-7259, 2004

17. Weiser MR: AJCC 8th Edition: Colorectal Cancer. Ann Surg Oncol 25: 1454-1455, 2018.

18. Punt CJA, Koopman M and Vermeulen L: From tumour heterogeneity to advances in precision treatment of colorectal cancer. Nat Rev Clin Oncol 14: 235-246, 2017.

19. Sveen A, Kopetz S and Lothe RA: Biomarker-guided therapy for colorectal cancer: Strength in complexity. Nat Rev Clin Oncol 17: 11-32, 2020

20. SargentD, Sobrero A, Grothey A,O'Connell MJ,Buyse M, Andre T, Zheng Y, Green E, Labianca R, O'Callaghan C, et al: Evidence for cure by adjuvant therapy in colon cancer: Observations based on individual patient data from 20,898 patients on 18 randomized trials. J Clin Oncol 27: 872-877, 2009.

21. Konishi T, Shimada Y, Hsu M, Tufts L, Jimenez-Rodriguez R, Cercek A, Yaeger R, Saltz L, Smith JJ, Nash GM, et al: Association of preoperative and postoperative serum carcinoembryonic antigen and colon cancer outcome. JAMA Oncol 4: 309-315, 2018.

22. Yakabe T, Nakafusa Y, Sumi K, Miyoshi A, Kitajima Y, Sato S, Noshiro $\mathrm{H}$ and Miyazaki K: Clinical significance of CEA and CA19-9 in postoperative follow-up of colorectal cancer. Ann Surg Oncol 17: 2349-2356, 2010.

23. Fletcher RH: Carcinoembryonic antigen. Ann Intern Med 104: 66-73, 1986.

24. Das PM and Singal R: DNA methylation and cancer. J Clin Oncol 22: 4632-4642, 2004.

25. Bartel DP: MicroRNAs: Target recognition and regulatory functions. Cell 136: 215-233, 2009.

26. Lin S and Gregory RI: MicroRNA biogenesis pathways in cancer. Nat Rev Cancer 15: 321-333, 2015.

27. Ding N, Sun X, Wang T, Huang L, Wen J and Zhou Y: miR 378a 3p exerts tumor suppressive function on the tumorigenesis of esophageal squamous cell carcinoma by targeting Rab10. Int J Mol Med 42: 381-391, 2018.

28. Li H, Dai S, Zhen T, Shi H, Zhang F, Yang Y, Kang L, Liang Y and Han A: Clinical and biological significance of miR-378a-3p and miR-378a-5p in colorectal cancer. Eur J Cancer 50: 1207-1221, 2014.

29. Valentino A, Calarco A, Di Salle A, Finicelli M, Crispi S Calogero RA, Riccardo F, Sciarra A, Gentilucci A, Galderisi U, et al: Deregulation of MicroRNAs mediated control of carnitine cycle in prostate cancer: Molecular basis and pathophysiological consequences. Oncogene 36: 6030-6040, 2017.

30. Zhang G-J, Zhou H, Xiao H-X, Li Y and Zhou T: MiR-378 is an independent prognostic factor and inhibits cell growth and invasion in colorectal cancer. BMC Cancer 14: 109, 2014.

31. Winder T and Lenz H-J: Vascular endothelial growth factor and epidermal growth factor signaling pathways as therapeutic targets for colorectal cancer. Gastroenterology 138: 2163-2176, 2010.

32. Yang LC, Lai CY, Hsieh CC and Lin WC: Natural killer cell-mediated anticancer effects of an arabinogalactan derived from rice hull in CT26 colon cancer-bearing mice. Int J Biol Macromol 124: 368-376, 2019.

33. Paschos KA, Canovas D and Bird NC: The role of cell adhesion molecules in the progression of colorectal cancer and the development of liver metastasis. Cell Signal 21: 665-674, 2009.

34. Moradi-Marjaneh R, Hassanian SM, Fiuji H, Soleimanpour S, Ferns GA, Avan A and Khazaei M: Toll like receptor signaling pathway as a potential therapeutic target in colorectal cancer. J Cell Physiol 233: 5613-5622, 2018.

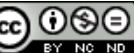

This work is licensed under a Creative Commons Attribution-NonCommercial-NoDerivatives 4.0 International (CC BY-NC-ND 4.0) License. 